\title{
FLAT CORE PROPERTIES ASSOCIATED TO THE $p$-LAPLACE OPERATOR
}

\author{
SHOSHANA KAMIN AND LAURENT VERON
}

(Communicated by Barbara L. Keyfitz)

\begin{abstract}
We study the formation of a flat hat pattern in the profile of the positive solution of an equation of the type: $\varepsilon \Delta_{p} u-u^{p-1}(1-u)^{\theta}=0 \quad(0<$ $\theta<p-1)$ in a bounded domain $\Omega$. When $\varepsilon$ tends to $0^{+}$, the growth of the zone where $u=u_{\varepsilon}$ takes the value 1 in $\Omega$ is studied.
\end{abstract}

\section{INTRODUCTION AND STATEMENT OF THE RESULTS}

This paper deals with the study of the limit behaviour when $\varepsilon$ tends to $0^{+}$ of the shape of the positive solution $u=u_{\varepsilon}$ of the problem

$$
\begin{array}{rlrl}
-\varepsilon \Delta_{p} u+f(u) & =0 & & \text { in } \Omega, \\
u=0 & & \text { on } \partial \Omega,
\end{array}
$$

where $\Omega$ is a connected, bounded open subset of $\mathbb{R}^{N}, N \geq 2$, with a $C^{2}$ boundary $\partial \Omega ; \Delta_{p}$ is the $p$-Laplace operator defined by

$$
\Delta_{p} u=\operatorname{div}\left(|\nabla u|^{p-2} \nabla u\right)
$$

with $p>1$; and $f$ is continuous with nonpositive values. Such a problem appears when studying the stationary states of a strongly nonlinear heat equation in an absorbing-reacting media (see [D] for physical examples and further references). The precise hypotheses on $f$ are the following:

(H1) $f$ is continuous on $[0, \infty)$ and $r \mapsto f(r) / r^{p-1}$ is increasing.

(H2) $\lim _{r \downarrow 0} f(r) / r^{p-1}=-1$.

(H3) There exist $C>0$ and $\theta \in(0, p-1)$ such that $\lim _{r \uparrow 1} f(r) /(1-r)^{\theta}$ $=-C$.

The specific phenomenon we shall study is the formation of a flat hat pattern inside $\Omega$, that is, a zone where $u$ takes the value 1 and the growth of this zone when $\varepsilon$ tends to 0 .

The typical example of a function $f$ satisfying (H1)-(H3) is $f(u)=u^{p-1}$ $u^{q}$, thus problem (1) becomes

$$
\begin{aligned}
-\varepsilon \Delta_{p} u & =u^{p-1}-u^{q} & & \text { in } \Omega, \\
u & =0 & & \text { on } \partial \Omega .
\end{aligned}
$$

Received by the editors May 6, 1991 and, in revised form, November 26, 1991.

1991 Mathematics Subject Classification. Primary 35J60, 35J70. 
If we set $\varepsilon=1 / \lambda$ and $u=v \varepsilon^{1 /(q+1-p)}$, then (3) reads as

$$
\begin{aligned}
-\Delta_{p} v & =\lambda v^{p-1}-v^{q} & & \text { in } \Omega, \\
v & =0 & & \text { on } \partial \Omega .
\end{aligned}
$$

The appearance of the flat zone for the solution of (4) for large $\lambda$ was first observed by Guedda and Veron. These authors in [GV] studied the structure of the set of solutions of the nonlinear eigenvalue problem

$$
\begin{aligned}
-\left(\left|v_{x}\right|^{p-2} v_{x}\right)_{x} & =\lambda|v|^{p-2} v-|v|^{q-1} v \text { in }(0,1), \\
v(0) & =v(1)=0
\end{aligned}
$$

It is proved in [GV] that for

$$
q>p-1>1
$$

and $\lambda$ large enough, the unique positive solution of (5) satisfies $v(x)=\lambda^{1 /(q+1-p)}$ for $x \in[x(\lambda), 1-x(\lambda)]$ where $x(\lambda)>0$ and $x(\lambda) \sim C \lambda^{-1 / p}$ at infinity. Another consequence described in $[\mathrm{GV}]$ is that for $\lambda$ large enough, the set of solutions $v$ of (5) with $k-1$ simple zeros on $(0,1)$ and $v_{x}(0)>0$ is homeomorphic to the $(k-1)$-dimensional unit cube. P. L. Lions asked one of the authors whether such phenomenon still existed for the $N$-dimensional case.

If we define

$$
\lambda_{1}=\min \left\{\int_{\Omega}|\nabla u|^{p} d x / \int_{\Omega}|u|^{p} d x: u \in W_{0}^{1, p}(\Omega) \backslash\{0\}\right\},
$$

it is a classical fact that under condition (6), for any $\lambda>\lambda_{1}$ there exists $v$ positive in $\Omega$ satisfying (4). As for problem (1) we know from [DS] that if $\varepsilon<1 / \lambda_{1}$ and $f$ satisfies $(\mathrm{H} 1),(\mathrm{H} 2)$, then there exists a unique $u-u_{\varepsilon}$ belonging to $C^{1}(\bar{\Omega})$ which is a positive in $\Omega$ solution of $(1)$. Moreover if $(\mathrm{H} 3)$ holds then $u$ takes its values in $[0,1]$. If we define

$$
\mathbf{\Omega}_{\lambda}=\left\{x \in \Omega: v(x) \equiv \lambda^{1 /(q+1-p)}\right\}
$$

then $\Omega_{\lambda}$ is a compact, possibly empty, subset of $\Omega$. We have the following answer to Lions's question

Theorem 1. Assume (6), $\lambda>\lambda_{1}, v$ is the positive solution of (4), and $\Omega_{\lambda}$ is defined by (8). Then there exists $\lambda^{*}=\lambda^{*}(\Omega, p, q)>\lambda_{1}$ such that:

(i) if $\lambda<\lambda^{*}$ the set $\Omega_{\lambda}$ is empty;

(ii) if $\lambda \geq \lambda^{*}$ the set $\Omega_{\lambda}$ is not empty and

$$
\operatorname{dist}\left(\Omega_{\lambda}, \partial \Omega\right) \leq C \lambda^{-1 / p}
$$

where $C=C(\Omega, p, q)>0$.

Theorem 1 is a consequence of

Theorem 2. Assume (H1)-(H3) with $p>1$. Then for $\varepsilon>0$ small enough the coincidence set $\Omega_{\varepsilon}$ of the solution $u$ of (1) defined by

$$
\Omega_{\varepsilon}=\{x \in \Omega: u(x)=1\}
$$

is not empty and there exists a constant $C>0$ such that

$$
\operatorname{dist}\left(\Omega_{\varepsilon}, \partial \Omega\right) \leq C \varepsilon^{1 / p} .
$$


Proofs OF THE RESULTS

We first extend the function $f$ on $(-\infty, 0)$ such that the resulting function defined on $R$ is a continuous odd function. This function is still denoted by $f$.

Lemma 1. Let $w_{1}$ and $w_{2}$ be two functions belonging to $C(\bar{\Omega}) \cap W^{1, p}(\Omega)$ and such that

$$
0=w_{1} \leq w_{2} \text { on } \partial \Omega
$$

and

$$
\begin{gathered}
w_{1} \leq w_{2}, \\
-\Delta_{p} w_{1}+f\left(w_{1}\right) \leq 0, \\
-\Delta_{p} w_{2}+f\left(w_{2}\right) \geq 0
\end{gathered}
$$

in $\Omega$. Then there exists a function $w$ in $C_{0}(\Omega) \cap W^{1, p}(\Omega)$ satisfying

$$
\begin{gathered}
w_{1} \leq w \leq w_{2}, \\
-\Delta_{p} w+f(w)=0
\end{gathered}
$$

in $\Omega$.

This result is due to Deuel and Hess [DeH] and extends previous results of Amann, Sattinger, and others (see $[A]$ for example).

Lemma 2. Let $w \in C(\bar{\Omega}) \cap W_{0}^{1, p}(\Omega)$ be a positive solution of (17) in $\Omega$. Then for $C>1$ (resp. $0<C<1$ ) we have

$$
-\Delta_{p}(C w)+f(C w) \geq 0 \quad\left(\text { resp. }-\Delta_{p}(C w)+f(C w) \leq 0\right)
$$

in $\Omega$.

Proof. For $C>1$ we have

$$
\Delta_{p}(C w)=C^{p-1} \Delta_{p} w=C^{p-1} f(w)=(C w)^{p-1} f(w) / w^{p-1} .
$$

From (H1) we have $f(w) / w^{p-1} \leq f(C w) /(C w)^{p-1}$, which yields (18). The same proof applies for $0<C<1$.

Lemma 3. Assume (Hi) $(\mathrm{i}=1,2,3)$ and let $u=u_{\varepsilon}$ be the positive solution of (9). Then $u_{\varepsilon}$ converges to 1 as $\varepsilon$ tends to 0 , uniformly on any compact subset $K$ of $\Omega$.

Proof. By the maximum principle, $u_{\varepsilon} \leq 1$ in $\bar{\Omega}$. The intent of this proof is to construct a subsolution $v$ of (9) with the form

$$
v=1-e^{-\psi / \varepsilon^{\prime}}, \quad \varepsilon^{\prime}=\varepsilon^{1 / p},
$$

with some $\psi>0$ in $\Omega$, vanishing on $\partial \Omega$; the function $\psi$ will be made precise later. Then

$$
\nabla v=\frac{1}{\varepsilon^{\prime}} e^{-\psi / \varepsilon^{\prime}} \nabla \psi
$$

and

$$
-\Delta_{p} v=\left(\varepsilon^{\prime}\right)^{-p} e^{-(p-1) \psi / \varepsilon^{\prime}}\left\{(p-1)|\nabla \psi|^{p}-\varepsilon^{\prime} \Delta_{p} \psi\right\},
$$

which yields

$$
-\varepsilon \Delta_{p} v+f(v)=\left[(p-1)|\nabla \psi|^{p}-\varepsilon^{\prime} \Delta_{p} \psi\right] e^{-(p-1) \psi / \varepsilon^{\prime}}+f(v) .
$$


Set $E_{1}=(p-1)|\nabla \psi|^{p}, E_{2}=-\varepsilon^{\prime} \Delta_{p} \psi$, and $y=e^{-\psi / \varepsilon^{\prime}}$. We claim that for $\varepsilon^{\prime}$ small enough

$$
E_{1}+E_{2} \leq-y^{1-p} f(1-y)
$$

For $\delta>0$ we define

$$
\begin{aligned}
& \Omega_{-}^{\delta}=\{x \in \Omega: y \leq \delta\}=\left\{x \in \Omega: \psi \geq \varepsilon^{\prime} \ln (1 / \delta)\right\}, \\
& \Omega_{+}^{\delta}=\{x \in \Omega: y>\delta\}=\left\{x \in \Omega: \psi<\varepsilon^{\prime} \ln (1 / \delta)\right\} .
\end{aligned}
$$

From (H3) $\lim _{y \downarrow 0^{+}}\left(-y^{-\theta} f(1-y)\right)=C$; henceforth there exists $\delta_{0} \in(0,1)$ such that $-y^{-\theta} f(1-y)>C / 2$ for $0<y<\delta_{0}$, which implies

$$
-\frac{1}{y^{p-1}} f(1-y)>\frac{C}{2 \delta^{p-1-\theta}} \quad \forall y \in\left(0, \delta_{0}\right)
$$

as $p-1-\theta>0$. We shall take $\psi=\phi_{1}^{p}$ where $\phi_{1}$ is the unique positive solution with upper bound 1 of

$$
\begin{aligned}
-\Delta_{p} \phi_{1} & =\lambda_{1} \phi_{1}^{p-1} & & \text { in } \Omega, \\
\phi_{1} & =0 & & \text { on } \partial \Omega .
\end{aligned}
$$

There exists $M>0$ such that for any $\varepsilon^{\prime} \in(0,1]$ we have

$$
\left.|(p-1)| \nabla \psi\right|^{p}-\varepsilon^{\prime} \Delta_{p} \psi \mid \leq M
$$

and there exists $\delta_{1} \in\left(0, \delta_{0}\right]$ such that for $\delta<\delta_{1}$

$$
(p-1)|\nabla \psi|^{p}-\varepsilon^{\prime} \Delta_{p} \psi \leq M \leq c / 2 \delta^{p-1-\theta}
$$

in $\Omega$, which implies that (22) holds in $\Omega_{-}^{\delta}$.

For the estimate in $\Omega_{+}^{\delta}$ note that there exist two positive constants $l(\delta)$ and $r(\delta)$ such that

$$
-f(1-y) \geq l(\delta)(1-y)^{p-1} \quad \forall y \in(\delta, 1)
$$

and, consequently,

$$
-f(1-y) \geq r(\delta)\left(\psi / \varepsilon^{\prime}\right)^{p-1}
$$

if $\delta<y \leq 1$ or $\psi / \varepsilon^{\prime}<\ln (1 / \delta)$; we used here that $\left(1-e^{-\rho}\right) / \rho$ is bounded below on $(0, \ln (1 / \delta))$. In order to have

$$
E_{1} \leq-f(1-y) / y^{p-1}
$$

in $\Omega_{+}^{\delta}$, it is sufficient to assure (with $y \leq 1$ ) that

$$
(p-1)|\nabla \psi|^{p} \leq r(\delta)\left(\psi / \varepsilon^{\prime}\right)^{p-1}
$$

or, equivalently,

$$
\left(\varepsilon^{\prime}\right)^{p-1}|\nabla \psi|^{p} \leq \frac{r(\delta)}{p-1} \psi^{p-1}
$$

As $\psi=\phi_{1}^{p}$ we have

$$
\left(\varepsilon^{\prime}\right)^{p-1}|\nabla \psi|^{p} \psi^{1-p}=\left(\varepsilon^{\prime}\right)^{p-1} p^{p}\left|\nabla \phi_{1}\right|^{p} .
$$

For $\delta \in\left(0, \delta_{1}\right)$ fixed, we can choose $\varepsilon_{0}^{\prime}>0$ such that $(31)$ holds for $0<\varepsilon^{\prime}$ $\leq \varepsilon_{0}^{\prime}$. 
For the remaining term we have

$$
\Delta_{p} \psi=\Delta_{p} \phi_{1}^{p}=p^{p-1}(p-1)^{2} \phi_{1}^{(p-1)^{2}-1}\left|\nabla \phi_{1}\right|^{P}-\lambda_{1} p^{p-1} \phi_{1}^{p(p-1)} .
$$

As $\partial \phi_{1} / \partial \nu<0$ on $\partial \Omega$ there exists a neighborhood $\mathbf{D}$ of $\partial \Omega$ such that

$$
(p-1)^{2}\left|\nabla \phi_{1}\right|^{p}>\lambda_{1} \phi_{1}^{p}
$$

in $\mathbf{D}$. For $\delta$ fixed in $\left(0, \delta_{1}\right)$ there exists $\varepsilon_{1}^{\prime} \in\left(0, \varepsilon_{0}^{\prime}\right)$ such that for any $\varepsilon^{\prime} \in\left(0, \varepsilon_{1}^{\prime}\right), \Omega_{+}^{\delta} \subset \mathbf{D}$. For such a limitation on $\varepsilon^{\prime}$ we have $\Delta_{p} \psi>0$ in $\Omega_{+}^{\delta}$, which implies

$$
E_{1}+E_{2} \leq-y^{1-p} f(1-y)
$$

in $\Omega_{+}^{\delta}$. Henceforth, with this restriction on $\varepsilon^{\prime}, v$ satisfies

$$
-\varepsilon^{\prime} \Delta_{p} v+f(v) \leq 0
$$

in $\Omega$ and $v$ vanishes on $\partial \Omega$. Now we compare $u$ and $v$. By Vazquez's maximum principle [V], $\partial u / \partial \nu<0$ on $\partial \Omega$; therefore, there exists $C>1$ such that $C u \geq v$ in $\Omega$. Using Lemmas 2 and 1 we get that there exists a solution $u^{*}$ of (9) such that $v \leq u^{*} \leq C u$. By uniqueness $u^{*}=u \geq v$. For any compact subset $K \subset \Omega$, there exists $\eta(K)>0$ such that $\psi \geq \eta(K)$ on $K$. Letting $\varepsilon$ tend to 0 implies the claimed result.

Proof of Theorem 2. Let $\tilde{u}=\tilde{u}_{\varepsilon}=1-u$. From (H3) there exists $\delta_{0}>0$ such that $-\tilde{u}^{-\theta} f(1-\tilde{u})>c / 2$ for $0<\tilde{u} \leq \delta_{0}$, which yields

$$
-\Delta_{p} \tilde{u}+\frac{c}{2} \tilde{u}^{\theta} \leq 0
$$

if $0<\tilde{u} \leq \delta_{0}$. For $\eta>0$ let $K_{\eta}$ be the subset of the $x$ 's in $\Omega$ such that $\operatorname{dist}(x, \partial \Omega) \geq \eta$. From Lemma 3 , for any $\delta \in\left(0, \delta_{0}\right)$ there exists $\varepsilon(\delta)>0$ such that for $0<\varepsilon<\varepsilon(\delta)$ we have

$$
\max \left\{\tilde{u}_{\varepsilon}(x): x \in K_{\eta}\right\}<\delta .
$$

Let $h=h_{\delta}$ be the solution of

$$
\begin{array}{rlrl}
-\Delta_{p} h+\frac{c}{2} h^{\theta} & =0 & & \text { in } B_{\eta}(0), \\
h=\delta & & \text { on } \partial B_{\eta}(0) .
\end{array}
$$

We know from Diaz-Herrero's paper [DH] (see also [D, p. 41]) that there exists $\delta>0$ such that $h_{\delta}(0)=0$. Let $x_{0} \in K_{2 \eta}$. By comparison, $\tilde{u}(x) \leq h_{\delta}\left(x-x_{0}\right)$ for $\left|x-x_{0}\right|<\eta$. Thus $\tilde{u}\left(x_{0}\right)=0$. Therefore $\tilde{u}(x) \equiv 0$ on $K_{2 \eta}$.

In order to obtain the final estimate we use a local scaling argument. As $\partial \Omega$ is $C^{2}$ there exists $\rho>0$ such that for any $a \in \partial \Omega$ the open ball with center $a-\rho \vec{\nu}_{a}$ and radius $\rho$ is included into $\Omega\left(\vec{\nu}_{a}\right.$ is the normal unit vector to $\partial \Omega$ at $a)$. As we already proved, there exists $\varepsilon_{1}>0$ such that the positive solution $z$ of

$$
\begin{aligned}
-\varepsilon_{1} \Delta_{p} z+f(z)=0 & \text { in } B_{\rho}(0), \\
z=0 & \text { on } \partial B_{\rho}(0),
\end{aligned}
$$

is such that $z(x) \equiv 1 \forall x \in B_{\rho / 2}(0)$. For $k>0$ the function $z_{k}$ defined by $z_{k}(x)=z(k x)$ satisfies

$$
\begin{aligned}
-\varepsilon_{1} k^{-p} \Delta_{p} z_{k}+f\left(z_{k}\right)=0 & \text { in } B_{\rho / k}(0), \\
z_{k}=0 & \text { on } \partial B_{\rho / k}(0)
\end{aligned}
$$


and is such that $z_{k}(x) \equiv 1 \forall x \in B_{\rho / 2 k}(0)$. For $0<\varepsilon<\varepsilon_{1}$ let $k$ be $\left(\varepsilon_{1} / \varepsilon\right)^{1 / p}$, $k>1$. For any $a \in \Omega$ such that $\operatorname{dist}(a, \partial \Omega) \geq \rho / k$ we can compare $u(x)$ and $z_{k}(x-a)$ in $B_{\rho / k}(a)$. By the same way as in the proof of Lemma 3, we use Lemmas 2 and 1 with $\alpha>0$ small enough. We get

$$
\alpha z_{k}(x-a) \leq u(x) \text { in } B_{\rho / k}(a),
$$

which implies

$$
z_{k}(x-a) \leq u(x) \text { in } B_{\rho / k}(a) .
$$

We deduce that $u \equiv 1$ in $B_{\rho / 2 k}(a)$, which implies (11).

Remark 1. It is clear that the coincidence set $\Omega_{\varepsilon}$ may be empty if $\varepsilon$ is too large. To have an estimate of this minimal $\varepsilon$ we can proceed as follows: let $d>0$ be the infimum of the distance of two hyperplanes that are parallel and such that $\Omega$ is contained into the strip limited by them. As the equation (9) is equivariant with respect to rotations and translations in $\mathbb{R}^{N}$, we can assume that

$$
\Omega \subset\left\{x=\left(x_{1}, x^{\prime}\right) \in \mathbb{R} \times \mathbb{R}^{N-1}: 0<x_{1}<d\right\} .
$$

Let $\zeta$ be the unique positive solution of

$$
\begin{gathered}
-\varepsilon\left(\left|\zeta_{x_{1}}\right|^{p-2} \zeta_{x_{1}}\right)_{x_{1}}+f(\zeta)=0 \quad \text { in }(0, d), \\
\zeta(0)=\zeta(d)=0 .
\end{gathered}
$$

It is clear that $\tilde{\zeta}(x)=\zeta\left(x_{1}\right)$ satisfies

$$
\begin{array}{rlrl}
-\varepsilon \Delta_{p} \tilde{\zeta}+f(\tilde{\zeta}) & =0 & & \text { in } \Omega, \\
\widetilde{\zeta} \geq 0 & & \text { on } \partial \Omega .
\end{array}
$$

As before there exists a solution $\tilde{u}$ such that for some $\alpha<1$

$$
\alpha u \leq \tilde{u} \leq \zeta
$$

and by uniqueness $\tilde{u}=u \leq \zeta$. If $0<\zeta<1$ in $(0, d)$ we deduce that the coincidence set $\Omega_{\varepsilon}$ is empty. In the particular case of equation (1) the coincidence set is empty if

$$
\begin{aligned}
& \lambda^{1 / p} d^{(q-1) /(q+1-p)} \\
& \quad<2 \int_{0}^{1}\left(\frac{q+1-p}{(p-1)(q+1)}+\frac{p}{(p-1)(q+1)} \sigma^{q+1}-\frac{\sigma^{p}}{p-1}\right)^{-1 / p} d \sigma
\end{aligned}
$$

[GV, Remark 2.3].

\section{ACKNOWLEDGMENTS}

This work was initiated while the second author was visiting Tel Aviv University in the framework of the Scientific Cooperation between France and Israel.

\section{REFERENCES}

[A] H. Amann, Fixed point equations and nonlinear eigenvalue problems in ordered Banach spaces, SIAM Rev. 18 (1976), 620-709.

[D] J. I. Diaz, Nonlinear partial differential equations and free boundaries, vol. I Elliptic equations, Research Notes in Math., vol. 106, Pitman, New York, 1985. 
[DeH] J. Deuel and P. Hess, A criterion for the existence of solutions of nonlinear elliptic boundary value problems, Proc. Roy. Soc. Edinburgh Sect. A 74 (1975), 49-54.

[DH] J. K. Diaz and M. A. Herrero, Estimates on the support of the solutions of some nonlinear elliptic and parabolic problems, Proc. Roy. Soc. Edinburgh Sect. A 89 (1981), 249-258.

[DS] J. I. Diaz and J. E. Saa, Existence et unicité de solutions positives pour certaines équations elliptiques quasilinéaires, C. R. Acad. Sci. Paris Sér. I Math. 305 (1987), 521-524.

[GV] M. Guedda and L. Veron, Bifurcation phenomena associated to the p-Laplace operator, Trans. Amer. Math. Soc. 310 (1988), 419-431.

[T] P. Tolksdorf, On the Dirichlet problem for quasilinear equations in domain with conical boundary points, Comm. Partial Differential Equations 8 (1983), 773-817.

[V] J. L. Vazquez, A strong maximum principle for some quasilinear elliptic equations, Appl. Math. Optim. 12 (1984), 191-202.

School of Mathematics, Raymond and Beverly Sackler faculty of Exact Sciences, TEL AVIV UNIVERSITY, ISRAEL

E-mail address: KAMIN@TAURUS.BITNET

Département de Mathématiques, Faculté des Sciences et Techniques, Parc de GrandMONT, 37200 TOURS, FRANCE

E-mail address: VERONL@FRUTRS51.BITNET 